British Journal of Education, Society \&
Behavioural Science
$18(4): 1-15,2016 ;$ Article no.BJESBS.30322
ISSN: 2278-0998
SCIENCEDOMAIN international
WwW.sciencedomain.org

\title{
Adaptive Functioning of Preschooler Children with Leukemia Post 1 Year of Therapies Compared with Sane Peers
}

\author{
Livia Taverna ${ }^{1}$, Marta Tremolada $^{2 *}$, Sabrina Bonichini ${ }^{2}$, Giuseppe Basso ${ }^{3}$ \\ and Marta Pillon ${ }^{3}$ \\ ${ }^{1}$ Faculty of Education, Free University of Bozen-Bolzano, Regensburger Allee 16, \\ 39042-Brixen-Bressanone, Italy. \\ ${ }^{2}$ Department of Developmental and Social Psychology, University of Padua, Via Venezia 8, \\ 35131 Padua, Italy. \\ ${ }^{3}$ Oncology Hematology Division, Department of Child and Woman Health, University-Hospital of \\ Padua, Via Giustiniani 2, 35127 Padua, Italy.
}

\section{Authors' contributions}

This work was carried out in collaboration between all authors. Author LT designed the study, collected data on sane children, supervised the work and wrote the first draft of the manuscript. Author MT collected data in the clinic, performed the statistical analysis, wrote the methods and results sections. Author SB supervised the data collection in the clinic and revised the final draft of the manuscript. Authors GB and MP collected the medical and psychological data in the hospital. All authors read and approved the final manuscript.

Article Information

DOI: 10.9734/BJESBS/2016/30322

Editor(s):

(1) Redhwan Ahmed Mohammed Al-Naggar, Population Health and Preventive Medicine, UniversitiTeknologi MARA,

Sungai Buloh, Selangor, Malaysia.

Reviewers:

(1) Mwiya Imasiku Liamunga, University of Zambia, Zambia. (2) Ghada Mohammad Hussein Abu Shosha, Zarqa University, Jordan.

(3) Takashi lkeno, National Center of Neurology and Psychiatry, Japan. (4) Ender Durualp, Ankara University, Turkey.

(5) P. Jayanthi, Tamilnadu Dr MGR Medical University, India. Complete Peer review History: http://www.sciencedomain.org/review-history/17084

Original Research Article

Received $2^{\text {nd }}$ November 2016

Accepted $26^{\text {th }}$ November 2016

Published ${ }^{\text {st }}$ December 2016

\section{ABSTRACT}

Background: Due to advances in chemotherapy and supportive care, about $83 \%$ of childhood cancer survivors aged 0-19 years will survive 5 years. More attention has been paid to understand the impact of cancer treatments on children's development, in particular with respect to late effects 
on academic, physical and social functioning, essential for optimization of long-term outcome. Scant literature addresses the functioning of children during acute treatment, mainly focusing on childhood cancer survivors.

Aims: This study was aimed at comparing the adaptive functioning in preschooler children with leukemia post 1 year of treatment and a control group of peers who had no history of serious illness. The link between medical factors and adaptive outcomes were investigated.

Study Design: Case control study.

Place and Duration of Study: Department of Child and Woman Health (Oncology Hematology Division), University of Padua, and pediatricians' ambulatories in the region of Trentino Alto Adige, between January 2007 and January 2008.

Methodology: The participants in this study included 48 leukemic children and their families, recruited at the Haematology-Oncologic Clinic of the Department of Child and Woman Health, University of Padua. Children's mean age was 52.35 months (SD $=12.85$, range $=23-72$ months), 22 males and 26 females. Most children had Acute Lymphoblastic Leukemia $(A L L)(N=44)$, while 4 had Acute Myeloid Leukemia (AML) with a mean days of hospitalization of 56.13 (SD = 49.36, range: 28-315). Sane peers $(\mathrm{N}=48)$ were recruited at pediatricians' ambulatories in the region of Trentino Alto Adige. Each family was interviewed about their children's adaptive functioning by Vineland Adaptive Behavior Scales.

Results: The two samples were homogeneous both for children's characteristics (age in months and gender) and for their parents' socio-demographic characteristics. Paired-sample t-tests revealed that parents of children with leukemia reported in their children significantly less adaptive functioning both composite and in three of the four domains: Communication, Socialization and Motor Abilities. The results of a series of ANCOVAs showed that the adaptive functioning in children with leukemia had the following at-risk factors: children aged 37-48 months, major days of hospitalization and the high risk therapy.

Conclusion: Future studies should understand better, with an ampler sample and using a multimethod approach, these lower adaptive outcomes in children with leukemia. These findings can guide specific interventions for parents and for children to support the potential developmental delay due to therapies, such as occupational therapy and socialization/educational programs during hospitalization, in occasion of day-hospital follow-ups and at school.

Keywords: Preschool; leukemia; adaptive functioning; developmental delay; sane peers.

\section{ABBREVIATIONS}

ALL : Acute Lymphoblastic Leukemia

AML : Acute Myeloid Leukemia

HSCT : Hematopoietic Stem Cell Transplantation

\section{INTRODUCTION}

\subsection{Children's Abilities and Adaptive Functioning during Cancer Treatments}

Children with leukemia could develop some delays of important skills, such as communication, daily living skills, socialization and motor abilities. The leukemia's therapies consist of intensive chemotherapy also directed on CNS, sometimes also Total Body Irradiation (TBI) and Hematopoietic Stem Cell Transplantation (HSCT) that could damage the normal development of the child, especially during the crucial preschool years. The literature focused principally on childhood cancer survivors' sequelae because, due to advances in chemotherapy and supportive care, about $82 \%$ of them in the age group 0-14 years have shown a dramatic increase in 5-year survival [1]. For this reason, more attention has been paid to understand the impact of treatments and interventions on children's development, particularly with respect to late effects on academic, physical and social functioning, essential for optimization of long-term outcome [2]. However, research on children with cancer has primarily focused on risks of neurocognitive deficits resulting from therapies directed to the central nervous system (e.g. irradiation, repetitive neurotoxic chemotherapy, or hematopoietic stem cell transplantation) and mainly focused on childhood cancer survivors [3]. Less is known about skills or abilities that enable a person to function adequately during cancer treatments and still in the maintenance phase of the therapy. Adaptive functioning reflects application of the children's abilities in daily life at an age appropriate level, and is strictly associated with the general concept of quality of life. Adaptive behaviors encompass a number of abilities and 
skills that allow a person to be included in her environment like communication, daily self-care, socialization skills and motor functioning. Basic motor, language and social adaptive competencies are acquired early in life reflecting maturation in interaction with formal or incidental learning [4]. As a result of this codetermination children develop adaptive skills in relation to their context of development, and with respect to opportunities to practice and application [5].

\subsection{Short and Late Effects of Therapies for Leukemia on Children's Development Skills}

\subsubsection{Physical and motor skills late effects}

Although a range of psychosocial and adaptive late-effects has been consistently documented in survivors of pediatric brain tumours [6,7], scant literature addresses the functioning of children during acute treatment. Research on acute adjustment to other pediatric cancers may provide a framework for understanding the impact of tumors more specifically [8]. Intrathecal methotrexate or triple intrathecal chemotherapy (methotrexate, cytarabine, and hydrocortisone) strategy in Acute Lymphoblastic Leukemia (ALL) had a beneficial effect for neuroendocrine aspects, while neurocognitive deficits were still observed 3 or more years after diagnosis, especially during the consolidation phase paralleling to a primarily calcification in the CNS [9].

Basic motor competencies are acquired early in life reflecting maturation in interaction with formal or incidental learning [4]. Chemotherapy could induce delayed skeletal muscle dysfunction in survivors of acute lymphoblastic leukemia [10]. In addition, pain and fatigue limit physical function [11], with gross and fine motor problems showed in leukemia survivors still also two years after cessation of treatment [12] and with significant and persistent visual-motor problems throughout the two years of therapies. These basic processing skills are necessary for the development of higher-level cognitive abilities, including nonverbal intelligence and academic achievement, particularly in arithmetic and written language [13].

\subsubsection{Communication skills late effects related to academic achievement}

Children's CNS therapies may affect the acquisition of new skills such as math and reading. Treatment that results in frequent and/or prolonged absence from school may be especially problematic for math learning [14]. Myelination occurring between the ages of 2-6 years coincides with a critical period of early language development [15] and white matter growth and maturation is important for cognitive growth [16]. No significant difference between children treated with intrathecal chemotherapy and controls were found when comparing change in performance scores from baseline measurement to 2 years post-baseline measurement performance scores [17], even if in another study this type of patients showed impaired expressive language skills than controls [18].

\subsubsection{Social skills late effects}

Children with ALL are often hospitalized for long periods because of their immunosuppressed status and because of the therapies. They are frequently forced to long periods in bed or in a restricted area (such as hospital room), where they are not allowed to meet friends or relatives. Fewer contacts with peers mean fewer opportunities to develop social skills [19], repeated school absence and long hospitalizations may cause delays in school learning [20] and a restricted academic success [21]. In the social health domain, children with ALL reported significantly lower abilities to maintain friendship and social competence, showing, when survivors, more compromised areas towards the relationships with their best friend [22]. Social skills were primarily associated with academic functioning [23] and neurocognitive deficits, specifically problems in verbal cognitive abilities and visual-motor integration skills, were significantly associated with poor psychosocial quality of life [24].

\subsubsection{Illness and treatment risk factors for neurocognitive impairments}

Patients that underwent the steroid therapy with dexamethasone were more at risk for neurocognitive late effects [25], performing more poorly on two of the four measures of academic achievement (i.e., reading comprehension and arithmetic calculation).

A few longitudinal studies were conducted and addressed child development from the first year of therapies to the off therapy time point. One example is the study of Jansen et al. [26] that showed no adverse effect of illness and psychological factors on IQ and 
neuropsychological functioning of patients recently diagnosed with ALL. Another prospective longitudinal study [27] confirmed that also at the end of the therapies the use of chemotherapy was not linked to major cognitive impairment, especially in school achievement. The cognitive impairments in the children with leukemia can be related to the hematopoietic stem cell transplantation (HSCT) [28] with IQ and adaptive behavior scores dropped significantly during the first year, but did not change in the successive follow-ups (from 1 year to 3 years). Also attentional dysfunctions are found in survivors of childhood ALL, especially in case of intensified treatment protocols [29].

\subsection{Study Aims and Hypotheses}

Findings from studies conducted during cancer treatment give disaccording results and need to be more carefully investigated. Neurocognitive deficits may not be evident in the period immediately following treatments, but may become evident later, for example with child's reentry to school. Comparing children with leukemia throughout the therapies with sane peers could be useful to identify children more at risk for developmental and adaptive delays. The present study is aimed at casting more light into the results from the scarce literature on children with leukemia during their maintenance phase of therapy, when they could partially re-entry to their daily routines life.

The research questions addressed are the following:

First, are the two matched samples homogeneous with respect to children's characteristics and parents' sociodemographic features?

Second, based on the literature on the short and long-term late effects, which are the types of adaptive functioning delays in preschool children with leukemia comparing with sane peers? Specifically, what is the situation for each domain: Communication, Daily Living skills, Socialization and Motor Abilities?

Third, which are the best illness predictive factors that influence adaptive behavior?

Basing upon the existing literature, we expect that children with leukemia under treatment have socialization problems related both to interpersonal relationships $[19,22]$ and to the respect of social and community rules, particularly due to their isolation experience. We also think that children under 6 years of age can have difficulties in their motor abilities $[10,13]$ proportionally to their days of hospitalization and to the type of therapeutic treatment. The only study assessing adaptive functioning in children with cancer adopting the Vineland Scales, showed a significant decrease in adaptive scores during the first year after the blood stem cell transplantation [28], so we expected HSCT to be a risk factor for a maladaptive functioning.

\section{MATERIALS AND METHODS}

\subsection{Participants}

One hundred and twenty-two families recruited at the Haematology-Oncologic Clinic of the Department of Child and Woman Health, University of Padua, agreed to participate to the research-clinical project entitled "Family factors predictive short and long term adaptation and quality of life in children with leukemia. A longitudinal study". The families were contacted by a clinical psychologist during the first hospitalization of their children, about one week after the diagnosis. Project aims were explained and informed consent was asked for. The parents were interviewed in a separate room of the Clinic by EFI-C [30]. A questionnaires battery was compiled throughout several established time points: second week after the diagnosis communication, 1 month later, 6 months later, 12 months later and 24 months later.

In this study the following inclusion criteria were considered: children aged from 2 to 6 years, with a diagnosis of Acute Lymphoblastic Leukemia (ALL) or Acute Myeloid Leukemia (AML); time of administration (12- months post diagnosis, when children are in the Maintenance phase of therapy; no history of neurodevelopmental disorders such as learning or sensory disabilities or other genetic syndromes.

After 12 months parents were interviewed adopting the Vineland Adaptive Behavior Scales. From the initial sample we took into consideration only the preschooler age group (23-74 months) which were 75 at this time point. Of these, 15 gave up the study for several reasons: Deceased or in a terminal condition $(\mathrm{N}=9)$, center changed or dropped off $(\mathrm{N}=6)$. The response rate attested to $92 \%$ excluding the deceased patients. Forty-eight sane peers were 
matched by age in months and gender with the remaining 60 patients for this study. Children's mean age was 52.35 months $(S D=12.85$, range = 23-72 months), 22 males and 26 females. Most children had Acute Lymphoblastic Leukemia (ALL) $(\mathrm{N}=44)$, while 4 had Acute Myeloid Leukemia (AML) with a mean days of hospitalization of $56.13(S D=49.36)$. Eleven children belong to the standard risk protocol, 32 intermediate risk and 5 followed the high risk protocol. All parents were Caucasian with a mean age of 36.81 years $(S D=6.93)$ and a mean of 12.33 years of schooling $(S D=3.61)$. Parents' incomes were mostly average (51.2\%), followed by an equally distribution between high (24.4\%) and low (24.4\%) for Italian norms, but above poverty. The average of job hours/weekly was mostly around $35-45$ (65.1\%), followed by the average of $25(14 \%)$, and of $5-15(9.3 \%)$, and over $50(2.3 \%)$. There is a part of parents temporarily relieved of their work or were housewives $(37.8 \%)$, a part of full time $(35.6 \%)$ and part-time $(22.2 \%)$ and also a percentage $(4.4 \%)$ of parents that lose their job. The number of children for each family was mostly two $(\mathrm{N}=$ $32)$, followed by one $(N=13)$ and by three $(\mathrm{N}=3)$.

Control group participants $(\mathrm{N}=48)$ met the following eligibility criteria: no history of lifethreatening, chronic illness or injury (for example, diabetes or severe asthma), neurodevelopmental disorders such as learning or sensory disabilities or other genetic syndromes. The control group of healthy peers consisted in those enrolled at paediatricians' ambulatories in the region of Trentino Alto Adige.

We created 48 patient-healthy peer couples with the same gender and age in months. Mean age of clinic group was 52.35 months $(S D=12.79$; range 23-72) and mean age of control group was $54.04(\mathrm{SD}=12.79$; range 24-71).

\subsection{Procedure}

Ethics approval was obtained from the local committees. The families were contacted by a clinical psychologist during the first hospitalization of the children, about one week after the diagnosis. Project aims were explained and informed consent was asked for. Informal contacts with the participants were kept up on a daily basis, to provide support and motivation for the project. The participants were informed that they were free to drop out at any moment of the study. Each family was contacted again 12 months later, when the VABS [31,32] was administered. The assessments were carried out at the Day Hospital or in the library of the Clinic. The psychologist remained constantly in touch with the child and the family for the duration of the study with frequent telephone contacts and direct contacts during the $\mathrm{DH}$ check-ups. Before the assessments, the psychologist contacted by telephone the parents to agree about the meetings. Also medical and socio-demographic information were collected.

\subsection{Instruments}

\subsubsection{Vineland adaptive behaviour scales (VABS) [31,32]}

Adaptive functioning was assessed with the Vineland Adaptive Behavior Scale (VABS) which is a psychometrically validated parent interview administered by a trained psychological examiner. The VABS provides norm-referenced scores on a range of adaptive behaviors at developmental levels from birth through adulthood.

The VABS are useful in assessing an individual's daily functioning throughout several domains of adaptive functioning (personal and social). They can be used as an evaluation and diagnostic tool for individuals who can have mentally delays. They can also be used to develop individual educational, rehabilitative, and treatment programs and can monitor progress during such a program. Finally, the VABS can be used in research in which the development and functioning are investigated.

The VABS Interview Edition, Expanded Form, is administered to a parent or caregiver in a semistructured interview format. It has 540 items and it yields a more comprehensive assessment of adaptive behavior giving a systematic basis for preparing individual educational, rehabilitative, or treatment programs. It is organized around four adaptive behavior domains: Communication, Daily Living Skills, Socialization, and Motor Abilities. In the Expanded Form, items are grouped in clusters, which are organized in developmental order under sub-domains that make up the domains. Administration time for the interview is around 60 minutes.

The Communication domain is comprised of three sub-domains: Receptive, Expressive, and Written Language. The Daily Living Skills scale includes the Personal, Domestic, and Community 
sub-domains. The Socialization scale consists of Interpersonal, Play and Leisure, and Coping Skills sub-domains. The Motor Abilities scale includes Gross and Fine motor abilities (Fig. 1).

Studies confirming the reliability and validity of the VABS have solidified this measure as one of the most widely used assessments of adaptive behavior [33].

Each item is rated "2" (behavior is usually or habitually performed), "1" (sometimes or partly performed), or " 0 " (never performed). In addition, there is a code ("N") for cases when the child has never had the opportunity to perform the activity and a code ("DK") to use when the caregiver does not know if the child performed the activity.
The manuals provide users with instructions for scoring caregiver responses. Domain and subdomain raw scores are obtained by summing the numerical values of the responses. The sum of the domain standard scores is used to obtain the composite standard score. Psychometric properties for normal population and for Expanded Form in the Italian standardization version are good, dealing with reliability [32].

\subsubsection{Socio-demographic and medical data}

Each parent filled in a socio-demographic questionnaire with inquiries into their highest year of schooling, their education, their perceived economic situation, their type of home situation, their romantic relationship and their type of employment.

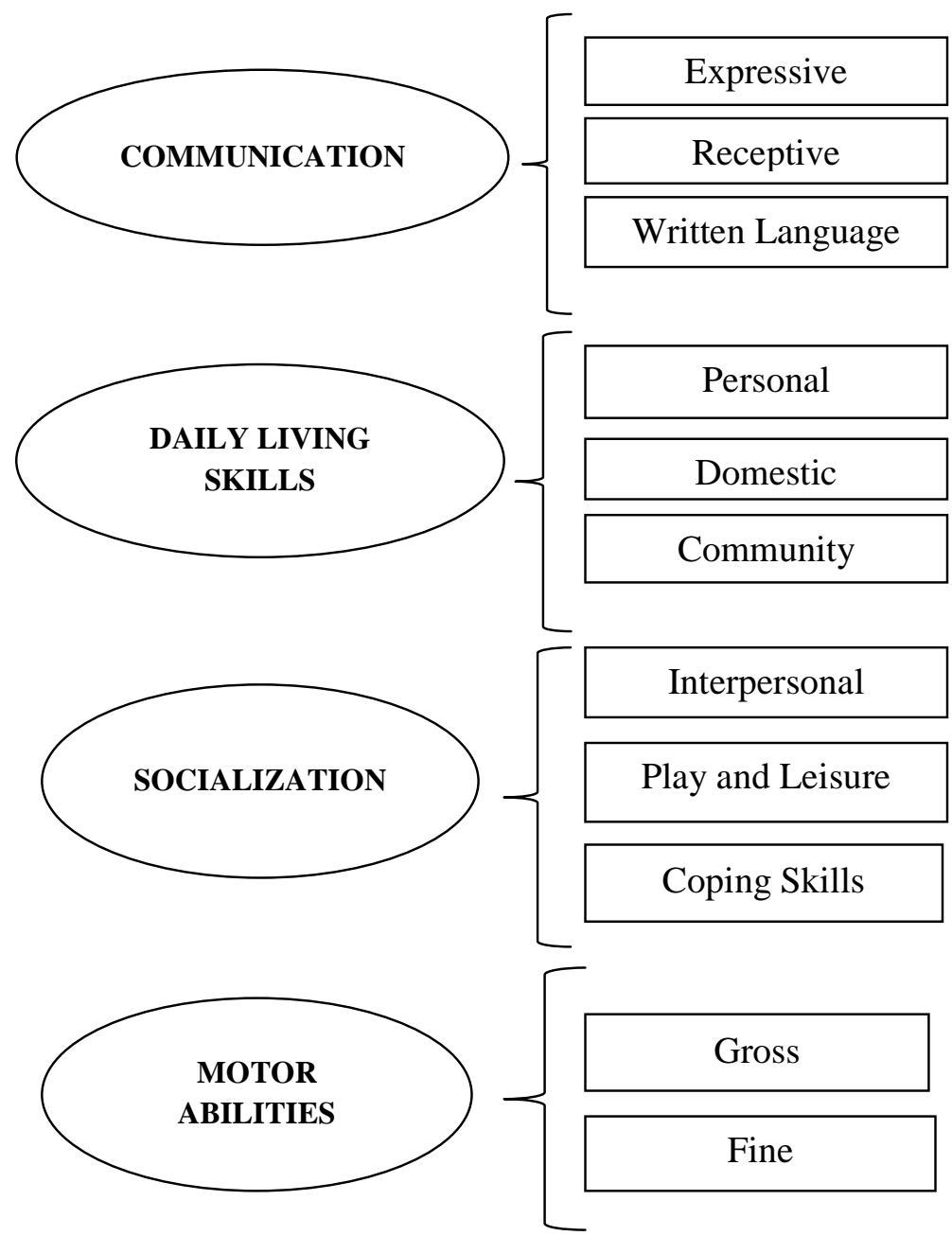

Fig. 1. Vineland' scales and subscales 
The oncologists who followed the patients extracted the necessary data from the patient's medical records. The medical data extrapolated from the records included: date of diagnosis, type of leukemia, therapy protocol risk band (SR, MR, $\mathrm{HR}$ ), age at diagnosis, HSCT (yes/no), toxicity effects of therapies (3rd and 4th grades).

\subsection{Statistical Methods}

We run descriptive statistics to show the Child's Adaptive Behavior Scores post 1 year of treatment, specifically the global adaptive behavior score and the scores related to each subscale (Communication, Daily Living Skills, Socialization, Motor Abilities).

A Chi-Square test was used to estimate the possible socio-demographic differences between the two samples (clinic and control) to better understand the comparability of the samples. The two groups were matched along characteristics of gender and age in months. Descriptive measures of central tendency and variability were computed for all relevant variables and comparisons between the two groups were run. Inferential comparisons between cancer survivors and control samples using a paired-sample t-test and controlling the effect size $d$ were done.

Then a series of ANCOVAS were run to identify predictors of adaptive functioning domains in children with leukemia. The independent fixed variables inserted in the model were: HSCT (presence vs absence), risk band (SR, MR, HR). The covariates inserted in the model were: Days of hospitalization, toxicities sum score. The dependent variables, inserted one by one, were the Adaptive functioning domains (Adaptive composite, Communication, Daily Living Skills, Socialization, Motor Abilities). Statistical significance was evaluated at the nominal $P=.05$ level, with adjustments for multiple comparisons, after controlling the normal distribution of the test scores and the homogeneity of variances. All data were analysed using SPSS version 18 (SPSS Inc., Chicago, IL).

\section{RESULTS AND DISCUSSION}

\subsection{Demographic Comparability and Differences between Family Characteristics of Children with Leukemia and of Sane Peers}

There were no significant socio-demographic differences between families of leukemia children and matched families of sane peers with respect to age and educational level of parents and to number of family members. The two samples were homogeneous both for children's characteristics (age in months and gender) and for their parents' socio-demographic features.

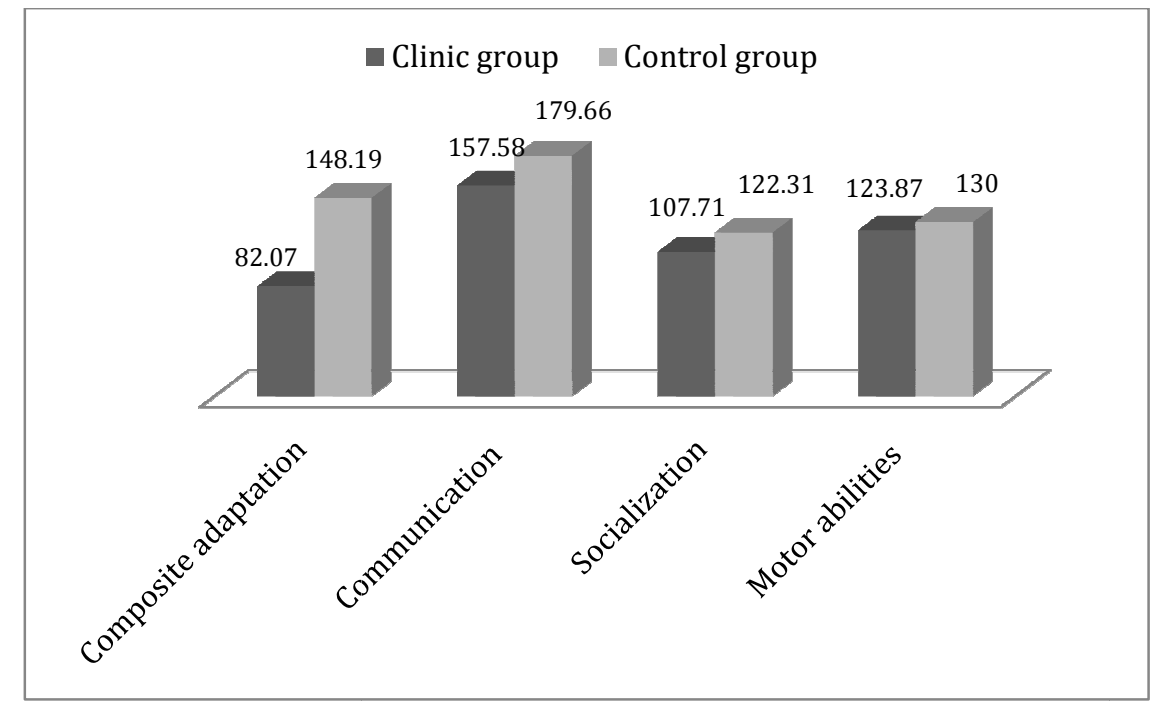

Fig. 2. VABS adaptive functioning domains' scores comparing children with leukemia post 1 year of treatment and controls 


\subsection{Adaptive Functioning between Children with Leukemia and Sane Peers}

The parents of children with leukemia reported in their children a significantly lower level of adaptive functioning both composite ( $\mathrm{t}_{47}=-9.37$; $\mathrm{p}=.001)$ and in three of the four domains: Communication (t $47=-9.37 ; P=.001$ ), Socialization (t ${ }_{47}=-5.52 ; P=.001$ ) and Motor Abilities ( $\mathrm{t}_{47}=-3.72 ; P=.001$ ) (Fig. 2).

The specific subdomains in which children with leukemia obtained lower scores in their adaptive functioning were specified below. For Communication domain, the subdomains Receptive ( $\mathrm{t}_{47}=-5.40 ; P=.001$ ) and Expression (t $\left.\mathrm{t}_{47}=-5.42 ; P=.001\right)$. Socialization subdomains reported for children with leukemia were all significantly lower: Interpersonal relationships (t $47=-3.73 ; P=.001)$, Play and Leisure $\left(\mathrm{t}_{47}=-\right.$ $3.42 ; P=.001)$ and Coping skills $\left(\mathrm{t}_{47}=-2.62 ; P\right.$ $=.01)$. The two Motor Abilities subscales obtained both lower scores: Gross ( $\mathrm{t}_{47}=-2.38$; $P=.002)$ and Fine $\left(\mathrm{t}_{47}=-1.93 ; P=.05\right)$ (Table 1 and Fig. 3).

\subsection{Adaptive Functioning between Children with Leukemia and Sane Peers by Age Groups}

We divided the children into three age groups: 23-36 months $(\mathrm{N}=5)$, 37-48 months $(\mathrm{N}=12)$, 4971 months $(\mathrm{N}=31)$ to have the adaptive functioning evaluation by children's age. We run the paired-sample t-test in each age

Table 1. Adaptive functioning in children with leukemia and controls

\begin{tabular}{|c|c|c|c|c|c|c|c|c|}
\hline & \multicolumn{2}{|c|}{$\begin{array}{l}\text { Children with } \\
\text { leukemia }(\mathrm{N}=48)\end{array}$} & \multicolumn{2}{|c|}{$\begin{array}{c}\text { Controls } \\
(\mathrm{N}=48)\end{array}$} & \multirow[t]{2}{*}{$\mathbf{N}$} & \multirow[t]{2}{*}{$\mathbf{t}$} & \multirow[t]{2}{*}{$\boldsymbol{P}$} & \multirow[t]{2}{*}{ d } \\
\hline & Mean & SD & Mean & SD & & & & \\
\hline Adaptive functioning composite & 82.07 & 51.22 & 148.19 & 25.51 & 48 & -9.37 & .0001 & 1.54 \\
\hline Communication & 157.58 & 40.72 & 179.66 & 28.19 & 48 & -5.52 & .0001 & .58 \\
\hline Receptive & 39.18 & 4.27 & 42.35 & 2.25 & 48 & -5.40 & .001 & .89 \\
\hline Expressive & 112.93 & 34.74 & 132.22 & 23.46 & 48 & -5.42 & .001 & .61 \\
\hline Socialization & 107.70 & 30.41 & 122.31 & 96.98 & 48 & -3.72 & .001 & .51 \\
\hline Interpersonal Relationship & 51.79 & 11.70 & 57 & 9.19 & 48 & -3.73 & .001 & .49 \\
\hline Play and Leisure & 42.54 & 12.35 & 48.50 & 13.35 & 48 & -3.42 & .001 & .46 \\
\hline Coping Skills & 12.43 & 8.14 & 16.81 & 9.24 & 48 & -2.62 & .01 & .50 \\
\hline Motor Abilities & 123.87 & 23.06 & 130 & 13.75 & 48 & -2.36 & .02 & .30 \\
\hline Gross & 74.54 & 11.89 & 77.81 & 6.10 & 48 & -2.38 & .02 & .60 \\
\hline Fine & 49.33 & 12.25 & 52.18 & 8.18 & 48 & -1.94 & .05 & .26 \\
\hline
\end{tabular}

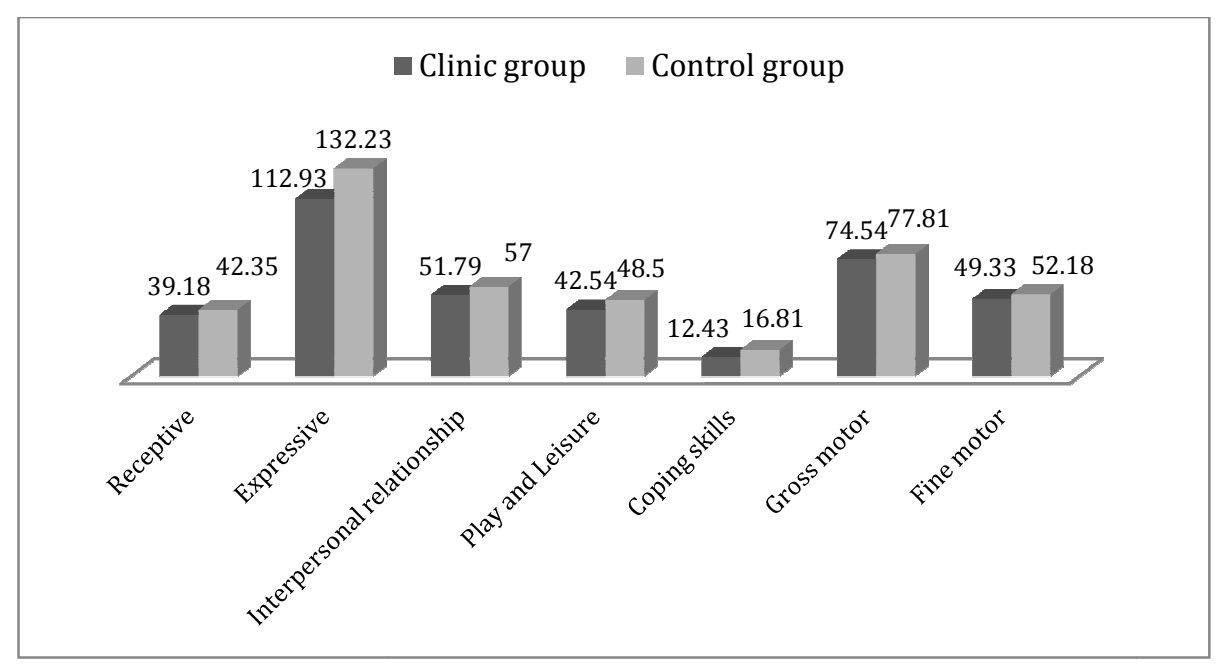

Fig. 3. VABS adaptive functioning subdomains' scores comparing children with leukemia post 1 year of treatment and controls 
group. A significant difference between clinic and control group is obtained for Adaptive functioning composite of children aged 37-48 months $\left(\mathrm{t}_{11}=-\right.$ $5.57 ; P=.001 ; \mathrm{d}=2.02)$ and those aged $49-71$ months ( $\mathrm{t}_{30}=-7.40 ; P=.001 ; \mathrm{d}=1.64$ ) with the clinic group showing lower scores than the control one. Communication abilities were recognized lower in the clinic group of children aged 37-48 months ( $\mathrm{t}_{11}=-3.82 ; P=.003 ; \mathrm{d}=$ 1.51) and in those aged 49-71 months ( $\mathrm{t}_{30}=-$ 4.61; $P=.001 ; d=1.03)$. Parents of children aged 37-48 months reported significantly lower score in Socialization ( $\mathrm{t}_{11}=-5.89 ; P=.001 ; \mathrm{d}=$ $1.85)$ and in the Motor Abilities ( $\mathrm{t}_{11}=-2.26 ; P=$ .004 ; $d=1.05$ ) (Figs. 4 and 5).

\subsection{Children with Leukemia Post 1 year of Treatment: Which are the Predictors of their Outcomes on Adaptive Functioning?}

A series of ANCOVA tests were run to understand the best illness predictors that favored a positive Adaptive functioning in children with leukemia post 1 year of treatment (Table 2).

The results showed the following at-risk categories: children with major days of hospitalization, under high risk therapy protocol and with a major therapy-toxicities.

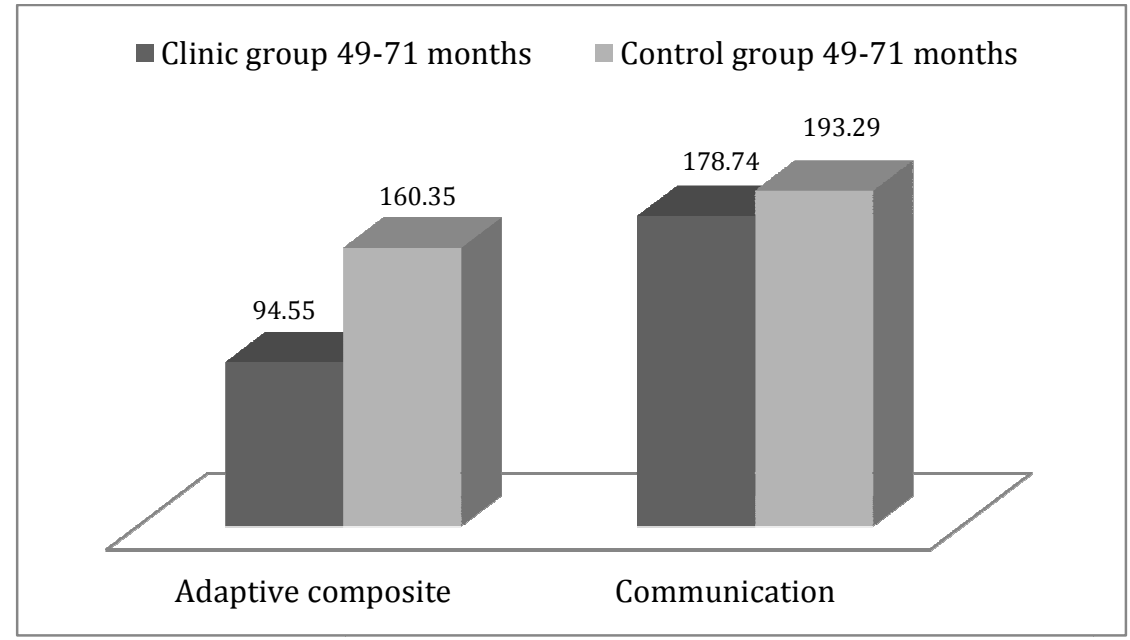

Fig. 4. VABS adaptive functioning domains' scores comparing children with leukemia aged 49-71 months post 1 year of treatment and matched controls

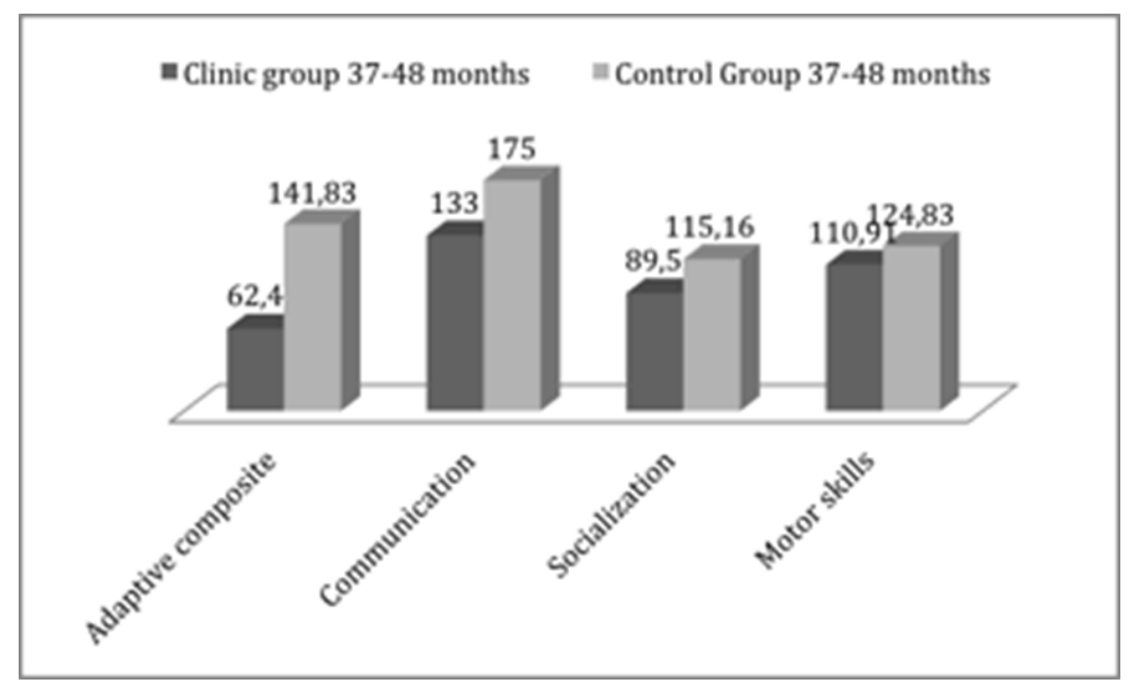

Fig. 5. VABS adaptive functioning domains' scores comparing children with leukemia aged 37-48 months post 1 year of treatment, and matched controls 
Taverna et al.; BJESBS, 18(4): 1-15, 2016; Article no.BJESBS.30322

Table 2. ANCOVAS to identify predictors of adaptive functioning domains and subdomains in children with leukemia post 1 year of treatment

\begin{tabular}{|c|c|c|c|c|c|c|c|c|c|c|}
\hline DV: Adaptive functioning & $\mathbf{N}$ & Source & df & $\mathbf{F}$ & $P$ value & $\eta_{p} 2$ & B & \multicolumn{3}{|c|}{ Estimated marginal means (DS error) } \\
\hline Adaptive composite & 48 & Risk band & 2 & 3.32 & .04 & .14 & .60 & $\begin{array}{l}S R \\
106.32(13.91)\end{array}$ & $\begin{array}{l}\text { MR } \\
74.02(8.82)\end{array}$ & $\begin{array}{l}H R \\
41.34(25.97)\end{array}$ \\
\hline Communication & 48 & Days of hospitalization & 1 & 11.20 & .002 & .21 & .90 & & & \\
\hline Daily Living Skills & 48 & Days of hospitalization & 1 & 7.85 & .008 & .16 & .78 & & & \\
\hline Socialization & 48 & Days of hospitalization & 1 & 7.36 & .01 & .15 & .75 & & & \\
\hline Motor skills & 48 & Days of hospitalization & 1 & 24.12 & .0001 & .36 & 1 & & & \\
\hline $\begin{array}{l}\text { DV: Adaptive functioning } \\
\text { subdomains }\end{array}$ & & Source & df & $\mathbf{F}$ & $P$ value & $\eta_{\mathrm{p}} 2$ & B & & & \\
\hline Receptive & 48 & Days of hospitalization & 1 & 4.47 & .04 & .12 & .54 & & & \\
\hline Expressive & 48 & Days of hospitalization & 1 & 6.99 & .01 & .17 & .73 & & & \\
\hline Written & 48 & Toxicities sum score & 1 & 5.06 & .03 & .13 & .59 & & & \\
\hline Personal & 48 & Days of hospitalization & 1 & 13.43 & .001 & .29 & .94 & & & \\
\hline Interpersonal relationship & 48 & Days of hospitalization & 1 & 4.96 & .03 & .13 & .58 & & & \\
\hline Play and Leisure & 48 & Days of hospitalization & 1 & 12.59 & .001 & .27 & .93 & & & \\
\hline Gross motor abilities & 48 & Days of hospitalization & 1 & 57.35 & .0001 & 63 & 1 & & & \\
\hline Fine motor abilities & 48 & Days of hospitalization & 1 & 11.98 & .002 & .26 & .91 & & & \\
\hline
\end{tabular}

Legend: $I V=$ risk band (SR = Standard-risk; $M R=$ Medium risk; HR=High risk), COV= days of hospitalization, toxicities sum score 


\subsection{Discussion}

Adaptive behavior is defined as the collection of the conceptual, social, and practical skills that have been learned by children in order to function in their everyday lives. Its assessment should relate to an individual's typical performance during daily routines and changing circumstances, not to maximum performance. The following common elements are found in definitions of adaptive behavior: The developmental nature of the behaviors; common dimensions of adaptive behavior; recognition of cultural influences and situational specificity; and emphasis on performance rather than skills or ability [34].

The adaptive behavior scales used in this study (VABS) emphasize the developmental qualities of the construct by providing age-based norms and items that cover a wide range of developmental activities such as: Cognitive, Communication and Academic Skills (i.e., conceptual skills); Social Competence Skills (i.e., social skills) and Independent Living Skills (i.e., practical skills) [35]. Some limitations in these adaptive skills may include: Not knowing how to perform the skill (acquisitions deficit); not knowing when to use learned skills (performance deficit); or other motivational factors that can affect the expression of skills (performance deficit). A child's strengths and limitations in adaptive skills should be documented within the context of community and cultural environments typical of the person's age peers [34] and this is the reason for using a control group to compare these adaptive skills.

What could happen when a child has to adapt to an important illness experience such as leukemia? Research on children with cancer has primarily focused on risks of neurocognitive deficits resulting from therapies directed to the central nervous system and mainly focused on childhood cancer survivors [3]. Less is known about skills or abilities that enable a person to function adequately during cancer treatments and still in the maintenance phase of the therapy.

In this study preschoolers that were actually under therapy for a leukemia showed important delays in their adaptive functioning and development. They were in an age stage where they have to discover the world and to reach/exceed their developmental tasks. Instead, they were limited by the illness and the chemotherapy treatments in developing a series of adaptive skills that could contribute to their personal growth. The major limitations of these children, compared with matched sane peers, were found in communication, socialization and motor skills, especially in children aged 37-48 months. At this age stage, children should discover the social world, learning the social rules, stay and play with the other peers, building the first important relationships. Paralleling, they should train their communication abilities and also motor experiences become always more complete and sophisticated. They generally begin to take confidence of their abilities. Leukemia experience probably arrest this normal development because they are forced to be isolated from peers and from siblings, they are not able to move freely and are obliged to stay mostly only with their parents and not with peers.

In the age range 49-71 months the main limitations were identified in the communication skills and in the adaptive skills composite scale. Probably, at this age the communication domain, specifically receptive and expressive functioning, become predominant and it is recognized more essential in the daily life and for the global adaptive functioning. The illness forces to isolation characterized by a lack of stimulation and interest in communication since daily experience is predominantly related to health conditions and to cancer therapies. Sometimes parents isolate their children even too much, due to the fact that children are immunosuppressed for the therapies and parents have a huge fear of possible germs infection.

The main findings of the studies on childhood cancer survivors showed that chemotherapy could induce delayed skeletal muscle dysfunction [10], with gross and fine motor problems also two years after cessation of treatment [12]. These basic processing skills are necessary to the development of higher-level cognitive abilities, including nonverbal intelligence and academic achievement, particularly in arithmetic and written language [13]. These basic difficulties summed with isolation from their peers could lead also to less interpersonal relationships [19, 22] and impair the development of communication abilities. Children's SNC therapies may affect the acquisition of new skills such as math and reading [14] and they can show impaired expressive language skills than controls [18].

At this purpose, it becomes fundamental to identify children more at risk for adaptive skills delays. 
The first risk factor is child's age, with children aged 37-48 months showing more delays in almost all the adaptive skills. The second factor is the number of days of hospitalization that could drastically dampen all the children's adaptive skills. Hospitalizations force children to stay at bed, limit their motor abilities and social contacts with peers or other family members, determine generally less stimulation because of their poor health conditions. The third factor is represented by the high risk therapy - with higher dosages of intrathecal chemotherapy and the presence of Hematopoietic stem cell transplantation - influencing negatively the global adaptive skills, as suggested in the studies on childhood cancer survivors [3].

Female gender was not identified as a risk factor as suggested in some studies on cognitive dysfunction in ALL paediatric patients [29]. There is no clear pathophysiological explanation for observed gender differences, and they are not reported in survivors of other tumors [36].

We also checked parents' symptomatology, measured by a battery of questionnaires not reported in this study, to verify if parents of children with leukemia could be a valid proxyreport or if their judgments could be influenced by their particular psychological status. Their psychological symptomatology was not related with their reports on children's adaptive functioning.

The limits of this study could be that the children were resident in a specific Italian region and are not very numerous, so that it is difficult to generalize these results. We have not any baseline measure of their adaptive skills before or close to the cancer diagnosis. However, it would have been impossible to assess the adaptive behavior prior to the illness, and, similarly, it would have been very difficult to have the parents' collaboration and their valid reports immediately after the diagnosis, when the therapies begin, as it is a very critical time.

Possible strengths of this study consist in the indepth interviews with parents of preschool patients during the maintenance phase of therapy, when the children can partially re-enter to their normal daily routines, meeting peers after the isolation and beginning primary school. This is the first study that focused specifically on adaptive skills in childhood leukemia in the preschool age and during therapies. Another strength is the use of a comparative control group of sane peers.

\section{CONCLUSION}

Parents reported lower levels of adaptive behaviour in their children under treatment for leukemia both in the composite score and in three of the four domains: Communication, Socialization and Motor Abilties. The adaptive functioning presented the following at-risk factors: age (37-48 months), higher number of days of hospitalization and higher risk therapy.

Future studies should understand better, with an ampler sample and using a multimethod approach, these lower adaptive outcomes in children with leukemia. These findings can guide specific interventions for parents and for children to fill in the potential gap in development due to therapies, such as promoting occupational therapy and socialization/educational programs during hospitalization, in occasion of day-hospital follow-ups and at school.

Some recommendations for health care providers dealing with preschool children could be given: it could be useful monitoring children's adaptive functioning throughout the therapies for leukemia and training the parents to carefully observe their children's behaviors. At this purpose it could be helpful to give specific developmental skills' kits (communication, motor and social functioning) to parents so that they could stimulate children on their specific developmental daily tasks. They could use it in the hospital in the form of play-tools under the supervision of the volunteers/educators trained by paediatric occupational therapists. Parents could also benefit feeling more useful in their parenting role, overcoming impotence related to the cancer experience and reducing the arousal of their emotional state. It is important to carry forward this project also at home and during the children's social re-entry creating a link with children's teachers and school experience.

\section{CONSENT}

All authors declare that written informed consent was obtained from the parents of the pediatric patients.

\section{ACKNOWLEDGEMENTS}

We greatly appreciate support and facilities received by the Faculty of Education at the Free 
University of Bozen-Bolzano. The first author is also grateful that the study was supported with a RTD-M/PSI-04 research position at the Free University of Bozen-Bolzano.

\section{COMPETING INTERESTS}

Authors have declared that no competing interests exist.

\section{REFERENCES}

1. AIRTUM Associazione Italiana Registro Tumori. Cancer survival in children and adolescents, report; 2012.

Available:http://www.registritumori.it/PDF/AIRTUM2012/EP37 1 s s1 1 85 1-7.pdf

2. Pinquart $M$, Teubert D. Academic, physical, and social functioning of children and adolescents with chronic physical illness: A meta-analysis. Journal of Pediatric Psychology. 2012;37(4):376-389. DOI: 10.1093/jpepsy/jsr106

3. Armstrong GT, Oeffinger KC, Chen $Y$, Kawashima T, Yasui $Y$, Leisenring $W$, et al. Modifiable risk factors and major cardiac events among adult survivors of childhood cancer. JCO. 2013;4:1-11.

DOI: 10.1200/JCO.2013.49.3205

4. Masten AS, Coatsworth JD. The development of competence in favorable and unfavourable environments: Lessons from research on successful children. American Psychologist. 1998;53(2):205220.

5. Trivers R. Social evolution. Menlo Park, CA: Benjamin Cummings; 1986.

6. Netson KL, Conklin HM, Wu S, Xiong X, Merchant TE. Longitudinal investigation of Adaptive functioning following conformal irradiation for pediatric craniopharyngioma and low-grade glioma. International Journal of Radiation Oncology, Biology and Physics. 2013;85(5):1301-1306.

DOI: 10.1016/j.jrobp.2012.10.031

7. Msall ME. Developing preschool surveillance tools for adaptive functioning: Lessons for neuro-oncology. European Journal of Pediatric Neurology. 2010; 14(XXX):368-379.

8. Robinson KE, Wolfe $\mathrm{KR}$, Yeates $\mathrm{KO}$, Mahone EM, Cecil KM, Ris MD. Predictors of adaptive functioning and psychosocial adjustment in children with pediatric brain tumor: A report from the brain radiation investigative study consortium. Pediatric
Blood and Cancer. 2015;62(3):509-516 . DOI: $10.1002 / p b c .25323$

9. Von Stackelberg A, Hartmann R, Buhrer C, Fengler R, Janka-Schaub G, Reiter A. High-dose compared with intermediatedose methotrexate in children with a first relapse of acute lymphoblastic leukemia. Blood. 2008;111(5):2573-2580.

DOI: 10.1182/blood-2007-07-102525

10. Scheede-Bergdahl C, Jagoe RT. After the chemotherapy: Potential mechanisms for chemotherapy-induced delayed skeletal muscle dysfunction in survivors of acute lymphoblastic leukemia in childhood. Front. Pharmacol; 2013.

Available:http://dx.doi.org/10.3389/fphar.20 13.00049

11. Garralda ME, Rangel L. Impairment and coping in children and adolescents fatigue syndrome: A comparative study with other pediatric disorders. Journal of Child Psychology and Psychiatry. 2004; 45(XXX):543-552.

DOI: 10.1111/j.1469-7610.2004.00244.X

12. Reinders-Messelink $H$, Schoemaker $M$, Goeken L, Van Den Briel M, Kamps W. Handwriting and fine motor problems after treatment for acute lymphoblastic leukemia. Handwriting and Drawing Research: Basic and Applied Issues. 1996; 215-225.

13. Hockenberry $M$, Krull $K$, Moore $K$, Gregurich MA, Casey ME, Kaemingk K. Longitudinal evaluation of fine motor skills in children with leukemia. J Pediatr Hematol Oncol. 2007;29:535-539.

DOI: 10.1097/MPH.0b013e3180f61b92

14. Barnes MA, Raghubar KP, English L, Williams JM, Taylor $\mathrm{H}$, Landry $\mathrm{S}$. Longitudinal mediators of achievement in mathematics and reading in typical and atypical development. J Exp Child Psychol. 2014;119:1-16.

DOI: 10.1016/j.jecp.2013.09.006

15. Bates E, Goodman J. On the emergence of grammar from the lexicon. In B. MacWhinney (Ed.), The emergence of language. Mahwah, NJ: Lawrence Erlbaum. 1999;29-79.

16. Mabbott DJ, Noseworthy M, Bouffet E, Laughlin S, Rockel C. White matter growth as a mechanism of cognitive development in children. Neuroimage. 2006;33(3):93646.

DOI: 10.1016/j.neuroimage.2006.07.024

17. Lewis FM, Perry ML, Murdoch BE. Longitudinal language outcomes following 
intrathecal chemotherapy for acute lymphoblastic leukaemia International Journal of Speech-Language Pathology. 2013;15(2):156-164.

DOI: $10.3109 / 17549507.2012 .684888$

18. Lewis FM, Perry ML, Murdoch BE, Docking KM. An investigation of general and high-level language skills in children treated with central nervous systemtargeted chemotherapy for acute lymphoblastic leukemia. Journal of Medical Speech-language Pathology. 2011;19(2): 27-36.

19. Reiter-Purtill J, Waller J, Noll RB. Empirical and theoretical perspectives on the peer relationships of children with chronic conditions. In: Roberts M.C., Steele, R., eds., Handbook of Pediatric Psychology. $4^{\text {th }}$ ed. New York: Guilford Press. 2009;672-688.

20. White NC. Educational related problems for children with cancer. Journal of Pediatric Oncology and Nursing. 2003;20: 50-55.

DOI: 10.1053/jpon.2003.81

21. Madan-Swain A, Katz ER, LaGory J. School and social reintegration after a serious illness or injury. In R.T. Brown (Ed.), Handbook of pediatric psychology in school settings. Mahwah, NJ: Erlbaum. 2004;637-655.

22. Katz LF, Leary A, Breiger D, Friedman D. Pediatric cancer and the quality of children's dyadic peer interactions. J. Pediatr. Psychol. 2011;36(2):237-247. DOI: 10.1093/jpepsy/jsq050

23. Newby WL, Brown RT, Pawletko TM, Gold $\mathrm{SH}$, Whitt JK. Social skills and psychological adjustment of child and adolescent cancer survivors. PsychoOncology. 2000;12(2):113-126.

DOI: $10.1002 /(\mathrm{SICl}) 1099-$

1611(200003/04)9:2<113::AID-

PON432>3.0.CO;2-F

24. Batson AK, Lottick NK, Neglia JP. The contribution of Neurocognitive functioning to quality of life after childhood acute lymphoblastic leukemia. Psycho-Oncology. 2014;23(6).

DOI: $10.1002 /$ pon.3470

25. Waber DP, Carpentieri SC, Klar N, Silverman LB, Schwenn M, Hurwitz CA, et al. Cognitive sequelae in children treated for acute lymphoblastic leukemia with dexamethasone or prednisone. Journal of Pediatric Hematology Oncology. 2000; 22:193-196.
26. Jansen NC, Kingma $A$, Tellegen $P$, van Dommelen RI, Bouma A, Veerman A, Kamps WA. Feasibility of neuropsychological assessment in leukaemia patients shortly after diagnosis: Directions for future prospective research. Archives of Disease in Childhood. 2005; 90:301-314.

27. Kingma A, van Dommelen RI, Mooyaart EL, Wilmink JT, Deelman BG, Kamps WA. No major cognitive impairment in young children with acute lymphoblastic leukemia using chemotherapy only: A prospective longitudinal study. Journal of Pediatric Hematology Oncology. 2002;24:106-114. DOI: 10.1038/sj.bmt.1700699

28. Kramer JH, Crittenden MR, DeSantes $\mathrm{K}$, Cowan MJ. Cognitive and adaptive behavior 1 and 3 years following bone marrow transplantation. Bone Marrow Transplantation. 1997;19:607-613.

29. Buizer Al, de Sonneville LM, van den Heuvel-Eibrink MM, Veerman AJ. Chemotherapy and attentional dysfunction in survivors of childhood acute lymphoblastic leukemia: Effect of treatment intensity. Pediatr Blood Cancer. 2005; 45(3):281-290.

DOI: $10.1002 / p b c .20397$

30. Tremolada M, Bonichini S, Weisner TS, Basso G, Pillon M. Parental narratives of health-related quality of life in children with leukaemia in the second week after diagnosis: The ecocultural family interviewcancer. Journal of Pediatric Oncology. 2013;1:119-128.

31. Sparrow S, Balla D, Cicchetti D. Vineland adaptive behavior scales interview edition: Survey form manual. Circle Pines, MN: American Guidance Service; 1984.

32. Balboni G, Pedrabissi L. Adattamento italiano delle vineland adaptive behavior scales (Sparrow S, Balla DA, Cicchetti DV). Firenze: O.S. Organizzazioni Speciali. 2003;1-268.

33. Sparrow S, Cicchetti D. Diagnostic uses of the vineland adaptive behavior scales. Journal of Pediatric Psychology. 1985;10: 215-225.

34. Harrison PL. Mental retardation, adaptive behavior assessment, and giftedness. In A.S. Kaufman (Ed.), Assessing adolescent and adult Intelligence. Boston: Allyn \& Bacon. 1990;533-585.

35. Thompson JR, McGrew KS, Bruininks RH. Adaptive and maladaptive behaviour: 
Functional and structural characteristics. In R. L. Schalock (Ed.), Adaptive behavior and its measurement: Implications for the field of mental retardation. Washington, DC: American Association on Mental Retardation. 1999;15-42.
36. Castellino SM, Ulrich NJ, Whelen MJ, Lange BJ. Developing interventions for cancer-related cognitive dysfunction in childhood cancer survivors, J Natl Cancer Inst. 2014;106(8):1-16. DOI: $10.1093 /$ jnci/dju186

(C) 2016 Taverna et al.; This is an Open Access article distributed under the terms of the Creative Commons Attribution License (http://creativecommons.org/licenses/by/4.0), which permits unrestricted use, distribution, and reproduction in any medium, provided the original work is properly cited.

Peer-review history:

The peer review history for this paper can be accessed here: http://sciencedomain.org/review-history/17084 